\title{
Size measurement of dry ice particles produced from liquid carbon dioxide
}

\section{AUTHOR(S):}

Liu, Yi-Hung; Calvert, Graham; Hare, Colin; Ghadiri, Mojtaba; Matsusaka, Shuji

\section{CITATION:}

Liu, Yi-Hung ...[et al]. Size measurement of dry ice particles produced from liquid carbon dioxide. Journal of Aerosol Science 2012, 48: 1-9

ISSUE DATE:

2012-06

URL:

http://hdl.handle.net/2433/158355

\section{RIGHT:}

(C) 2012 Elsevier Ltd.; This is not the published version. Please cite only the published version.; この論文は出版社版でありません。引用の際に は出版社版をご確認ご利用ください。 


\title{
Size measurement of dry ice particles produced from liquid carbon dioxide
}

\author{
Yi-Hung Liu ${ }^{a}$, Graham Calvert ${ }^{b}$, Colin Hare ${ }^{b}$, Mojtaba Ghadiri ${ }^{b}$, \\ Shuji Matsusaka*
}

a Department of Chemical Engineering, Kyoto University, Kyoto 615-8510, Japan

${ }^{\mathrm{b}}$ Institute of Particle Science and Engineering, University of Leeds LS2 9JT, UK

\begin{abstract}
The formation of dry ice particles in a jet flow has been studied experimentally. The particles were produced by rapid expansion of liquid carbon dioxide through a nozzle, based on the Joule-Thomson effect. Their size distribution was measured by a laser diffraction method. The experimental results showed that the primary dry ice particles ejected from the nozzle were about $1 \mu \mathrm{m}$ in mass median diameter. However, they grew initially in the jet flow and then became smaller due to sublimation. As a result, a bimodal size distribution was formed at increased distances from the nozzle outlet. The presence of a thermally insulated tube at the outlet of the expansion nozzle enhanced the agglomeration of the particles, whereby agglomerates of about $100 \mu \mathrm{m}$ in mass median diameter were recorded. The agglomeration process is considered to take place by the simultaneous processes of particle deposition and reentrainment; i.e. agglomerated particles are reentrained from the layer of dry ice particles deposited on the tube walls. The agglomerate size decreased with increasing flow velocity, due to the greater detachment force applied to the deposition layer. Therefore, the flow velocity was found to be an important parameter influencing the agglomeration of dry ice particles.
\end{abstract}

Keywords: Dry ice particle; Jet flow; Agglomeration; Particle size measurement; Deposition; Reentrainment.

* Corresponding author. E-mail addresses: matsu@ cheme.kyoto-u.ac.jp (S. Matsusaka). 


\section{Introduction}

Rapid expansion of carbon dioxide liquid through a nozzle can produce dry ice particles by the Joule-Thomson effect. The dry ice jet can be employed for dry cleaning. The advantage of dry cleaning methods is the simplicity of the system compared with wet cleaning methods, i.e. there is no drying after cleaning and no water processing. In 1986, Hoenig demonstrated dry ice blast cleaning, where dry ice particles penetrated the surface layer and removed the contaminants. Compared with an air jet, the impact of dry ice particles enhances the removal of particulate contaminants and can even remove organic solvents (Sherman et al., 1994). In addition to cleaning, dry ice jets have been employed for refrigeration. The gas-solids two phase flow in a refrigeration system was investigated by Yamaguchi et al. (2008). Moreover, granules have been produced by the rapid expansion of supercritical solutions (Matson \& Smith, 1989). A study using supercritical carbon dioxide was carried out to improve drug properties (Sonoda et al., 2009).

The size and amount of dry ice particles will have a great influence on the application. For example, higher concentrations of dry ice particles with sufficient inertia are more effective to remove contaminants. The formation of dry ice particles depends on temperature, pressure and the dimensions of the expansion chamber; thus the operation conditions must be precisely controlled. Swain et al. (1992) installed a thermally insulated chamber at the end of an expansion nozzle to make agglomerated dry ice particles. The agglomerated particles are useful for the dry ice blast cleaning because they do not sublimate as quickly as small primary particles, and larger particles have greater inertia.

Liu et al. (2010) ejected dry ice particles from an agglomeration chamber and observed the process by a high-speed microscope camera, showing that the agglomeration process depends on the temperature and velocity of the dry ice jet. This agglomeration process was explained by the simultaneous particle deposition and reentrainment in the tube chamber. Small dry ice particles deposited on the tube wall and formed a deposition layer; then, agglomerated dry ice particles were entrained from the layer into the jet flow. To expand on this work it would be beneficial to investigate the state of primary dry ice particles produced by expanding liquid carbon dioxide and analyzing the size of primary and agglomerated particles. 
This study focuses on the production of dry ice particles by expanding liquid carbon dioxide through an expansion nozzle. Additionally, the agglomeration of dry ice in a thermally insulated tube attached to the outlet of the nozzle is investigated. The size distribution and the amount of dry ice particles produced under various conditions are analyzed by a laser diffraction method and features of the dry ice jet are discussed in detail.

\section{Experimental apparatus and procedures}

Fig. 1 shows a schematic diagram of the experimental apparatus. High purity liquid carbon dioxide was used to produce dry ice particles. A flexible thermally insulated hose, $2 \mathrm{~m}$ long and $15 \mathrm{~mm}$ in inner diameter was connected between a high pressure carbon dioxide cylinder and an expansion nozzle. A pressure gauge was installed to measure the pressure of the carbon dioxide. In this study, the pressure was kept at 5.5 MPa. Fig. 2 shows the details of the nozzle. Three nozzle sizes were used for introducing the different flow rates of the dry ice jet. The mass flow rates for 0.1 , 0.2 and $0.5 \mathrm{~mm}$ nozzles were $0.2,0.5,2.9 \mathrm{~g} / \mathrm{s}$, respectively. To produce agglomerated dry ice particles, an acrylonitrile-butadiene-styrene (ABS) tube of 2, 4, or $6 \mathrm{~mm}$ inner diameter and $50 \mathrm{~mm}$ in length was attached to the outlet of the expansion nozzle.

The size distribution of the dry ice particles ejected from the nozzle or the tube was measured in-situ with a particle size analyzer (Spraytec, Malvern Instruments Inc.) based on the laser diffraction method. The laser beam, with a diameter of $20 \mathrm{~mm}$, was oriented perpendicular to the dry ice jet. In the measurements, beam steering, which means beam direction changing, was observed and affected the size distribution of dry ice particles. This occurs when a significant volume of jet gas or another gaseous phase apart from air is present in the measurement zone. Gases such as high pressure jets have a very different refractive index compared to air. This causes the laser beam to be defocused, leading to a high scattering signal on the first set of detector channels. Although this signal is not caused by particle scattering, it is interpreted as coarse particles. To overcome this problem, the detector range has to be limited. For the measurement of primary dry ice particles, the particle diameter range was limited to below $50 \mu \mathrm{m}$ because it was confirmed that primary dry ice particles were less than 
several tens of micrometer from observations using a high-speed microscope camera (Liu et al., 2010). For the measurement of agglomerates of dry ice particles, the range was limited to less than $300 \mu \mathrm{m}$. This range is determined by considering the appropriate particle diameter of the agglomerates with minimizing the extent of the noise caused by the beam steering. The attenuation, which is defined as the natural logarithm of the ratio of the intensity of the incident light to that of the light passing through the jet flow, was used for the analysis of the particle concentration in the dry ice jet. These calculations were carried out using a patented multiple scattering algorithm which corrects multiple scattering effects for light transmissions (Harvill et al., 1995; Harvill \& Holve, 1998). The accuracy of this measurement system was ensured at 96\% obscuration, in which our experiments were conducted. All the experiments were conducted under room conditions $\left(20 \pm 2{ }^{\circ} \mathrm{C} ; 1 \mathrm{~atm}\right)$.

\section{Results and discussion}

\subsection{Effect of nozzle diameter on production of dry ice particles}

The size distributions by mass of dry ice particles ejected from the expansion nozzles with different diameters were measured by the laser diffraction method. Fig. 3 shows the cumulative size distributions of dry ice particles ejected from the $0.5 \mathrm{~mm}$ nozzle at different distances, $d_{\mathrm{n}}$, from the nozzle outlet. The mass median diameter of the dry ice particles is about $1 \mu \mathrm{m}$ at $d_{\mathrm{n}}=10 \mathrm{~mm}$ and increases up to several micrometers as the measurement distance from the nozzle increases. This result indicates that the dry ice particles produced from the expansion nozzle grow in the jet flow. Furthermore, each size distribution appears to be a log-normal distribution.

Fig. 4 shows the cumulative particle size distributions of dry ice particles ejected from the $0.2 \mathrm{~mm}$ nozzle. The mass median diameter increases with increasing distance for $d_{\mathrm{n}}<40 \mathrm{~mm}$. This feature is similar to that of the $0.5 \mathrm{~mm}$ nozzle, shown in Fig. 3 . However, the particle size distribution obtained at $d_{\mathrm{n}} \geq 40 \mathrm{~mm}$ differs from the other distributions as it is skewed towards larger particles. In addition, the distribution is not unimodal but bimodal at $d_{n}=50 \mathrm{~mm}$. These results are probably caused by the following two competing phenomena; one is the growth of dry ice particles in the 
gaseous carbon dioxide jet, and the other is the sublimation from particle surfaces. For the smaller nozzle, the gas velocity is lower due to the lower mass flow rate, providing sufficient time for particles to grow larger than those of $0.5 \mathrm{~mm}$ nozzle. On the other hand, smaller particles will rapidly shrink due to sublimation. For the smaller nozzle diameter, the mass flow rate is reduced; hence it is difficult to maintain the temperature of the jet flow in the required range due to the heat exchange with the surrounding atmosphere. As a result, a smaller nozzle diameter will enhance the rate of sublimation.

Fig. 5 shows the results for the $0.1 \mathrm{~mm}$ nozzle. The particle size distribution obtained at $d_{\mathrm{n}}=5 \mathrm{~mm}$ looks like a log-normal distribution, but for $d_{\mathrm{n}} \geq 10 \mathrm{~mm}$, the size distribution changes from unimodal to bimodal. The particle diameter increases with increasing distance for $10 \leq d_{\mathrm{n}} \leq 20 \mathrm{~mm}$, but the trend reverses remarkably for $d_{\mathrm{n}}=25$ $\mathrm{mm}$, where a notable reduction is observed. The flow rate for the $0.1 \mathrm{~mm}$ nozzle is so small that sublimation occurs at a shorter distance from the nozzle outlet.

In order to observe more clearly the effect of the nozzle diameter and the distance from the nozzle outlet on the production of the dry ice particles, the mass median diameters of dry ice particles obtained under various conditions are plotted in Fig. 6. For the $0.5 \mathrm{~mm}$ nozzle diameter, the mass median diameter increases linearly with distance from 1 to $3 \mu \mathrm{m}$ for distances of 10 and $50 \mathrm{~mm}$, respectively. For the $0.2 \mathrm{~mm}$ nozzle diameter, the mass median diameter increases linearly with the distance in the range of $10 \leq d_{\mathrm{n}} \leq 40 \mathrm{~mm}$, but decreases at $d_{\mathrm{n}} \geq 40 \mathrm{~mm}$. For the $0.1 \mathrm{~mm}$ nozzle diameter, the increase in mass median diameter with distance is more erratic than for the other nozzle diameters, the mass median diameter decreases from $d_{\mathrm{n}}=20 \mathrm{~mm}$.

The amount of dry ice particles in the jet flow can be inferred from the attenuation determined by the laser diffraction method. Fig. 7 shows the relationship between the attenuation and the distance from the nozzle outlet for three nozzle sizes. For the 0.5 $\mathrm{mm}$ nozzle, the attenuation is high; this is because the mass flow rate of carbon dioxide is high and a large number of particles are produced. Since the mass flow rate decreases with decreasing nozzle diameter, the attenuation is lower for smaller nozzle diameters. The dry ice jet spreads radially downstream; thus, the attenuation decreases with the distance from the nozzle outlet. In addition, sublimation will cause a decrease in attenuation at larger distances. 


\subsection{Agglomeration of dry ice particles in the ABS tubes}

Liu et al. (2010) observed that dry ice particles formed agglomerates by depositing on the inner walls of a tube attached to the expansion nozzle. However, no measurement of agglomerate size was carried out then. Here in this work agglomerates were generated using different size tubes and their size distribution was analysed in-situ using the technique discussed in section 2.

Fig. 8 shows the cumulative size distribution of agglomerates of dry ice particles, obtained using the $0.5 \mathrm{~mm}$ nozzle and the $6 \mathrm{~mm}$ tube. The mass median diameter of the agglomerates is about $100 \mu \mathrm{m}$, which is much larger than that of the particles produced without the tube $(\sim 1 \mu \mathrm{m}$, see Fig. 3). Each distribution appears to be log-normal, and the dependence of the particle size distribution on the distance $d_{\mathrm{t}}$ from the tube outlet is found to be small. However, the mass fraction of smaller particles slightly decrease with the increase of distance $d_{\mathrm{t}}$. The smaller particles might further agglomerate or sublimate in the jet flow. The influence of sublimation is not seen in larger agglomerated particles.

Fig. 9 shows the results for the $0.1 \mathrm{~mm}$ nozzle and the $2 \mathrm{~mm}$ tube. The particle size distributions are similar to those in Fig. 8. In addition, many particle size measurements were carried out under different conditions, i.e. with $0.1,0.2$, and $0.5 \mathrm{~mm}$ nozzles, and the 2, 4, and $6 \mathrm{~mm}$ tubes, showing that the results were generally similar to each other although slight differences can be seen. We, also, observed the particles by a high-speed microscope camera. The observation results are reasonably in agreement with those for the particle size measurement.

Fig. 10 shows the results measured at $d_{\mathrm{t}}=40 \mathrm{~mm}$ using the $6 \mathrm{~mm}$ tube for the three investigated nozzle diameters. When the nozzle diameter is changed from 0.1 to 0.5 $\mathrm{mm}$, the mass median diameter varies from $100 \mu \mathrm{m}$ to $80 \mu \mathrm{m}$. As a large amount of liquid carbon dioxide is expanded from the $0.5 \mathrm{~mm}$ nozzle, the flow velocity in the tube is high. The variation of the mass median diameter is thought to be caused by the difference in the flow conditions. The effect of the flow conditions, including the residence time and the flow velocity in the tube, on the agglomeration is discussed in detail in Sections 3.3 and 3.4. 
Next, the amount of agglomerated dry ice particles was evaluated from the attenuation data. Fig. 11 shows the relationship between the attenuation and the distance from the tube outlet for different tube and nozzle diameters. The attenuation decreases with increasing distance from the tube outlet. This is because the dry ice jet expands radially downstream, therefore the particle concentration of dry ice particles decreases with the distance from the tube outlet. Furthermore, it is found that the attenuation increases with the increase of the nozzle and tube diameters. Since the mass flow rate of carbon dioxide increases with increase in nozzle diameter, the attenuation is high for a large nozzle diameter. As the tube diameter increases, the cross-section area of the jet flow increases. As a result, the number of particles detected in the cross-section area increases; thus, the attenuation increases.

\subsection{Effect of interparticle collision on agglomeration}

Dry ice particles can collide with each other in the flow and produce agglomerated particles by adhesion forces; therefore, the interparticle collisions are generally taken into account in the agglomeration process. The concentration of primary particles and the residence time in the tube are the dominant factors in determining agglomerate size. To estimate the fraction of primary particles remaining after the agglomeration process, $\gamma$, the following equation is applied (Friedlander, 2000).

$$
\gamma=\frac{n_{1, \mathrm{t}}}{n_{1,0}}=\frac{1}{\left(1+t / \tau_{\mathrm{B}}\right)^{2}},
$$

where $n_{1, \mathrm{t}}$ is the concentration of primary particles after the agglomeration process, $n_{1,0}$ is the initial concentration of primary particles, $t$ is the residence time and $\tau_{\mathrm{B}}$ is the characteristic time.

From the theoretical calculation based on the experimental conditions where the value of $t / \tau_{\mathrm{B}}$ is estimated to be less than 0.02 , the fraction of primary particles remaining is over $95 \%$. This indicates that most of the primary particles cannot agglomerate by interparticle collisions alone when the residence time is less than $30 \mathrm{~ms}$, which is calculated on the basis of the experimental results. In the experiment, a large number of agglomerates about $100 \mu \mathrm{m}$ in mass median diameter are produced and ejected from the tube. Therefore, additional factors affecting the agglomeration in the tube should 
be taken into consideration.

\subsection{Flow velocity in a tube}

In this work, interparticle collisions are not considered to be the main mechanism for agglomeration in the tube, as mentioned previously in $\S 3.3$. Agglomeration is believed to be due to particle deposition and reentrainment on the tube wall (Matsusaka \& Masuda, 1996; Adhiwidjaja et al., 2000; Theerachaisupakij et al., 2003; Liu et al., 2010). Primary particles deposit on the tube wall and form a deposition layer; then, agglomerates are entrained from the deposition layer by aerodynamic drag. The collision of the flowing particles to the deposition layer also enhances reentrainment. Under steady state operation particle deposition and reentrainment are in equilibrium. Since these phenomena depend on the gas flow velocity, this may be a key parameter for agglomeration.

The average gas flow velocities for nozzle diameters of $0.1,0.2$ and $0.5 \mathrm{~mm}$, tube diameters of 2, 4 and $6 \mathrm{~mm}$ and distances from the tube of 20, 40 and $60 \mathrm{~mm}$ have been obtained experimentally. Fig. 12 shows the effect of the flow velocity on the mass median diameter of the agglomerates. The mass median diameter tends to decrease with an increase in the flow velocity. As the detachment force acting on the deposition layer increases with the flow velocity, smaller agglomerates can be reentrained by this mechanism. Therefore, the experimental relationship is reasonably good, and the gas flow velocity will be an important parameter to predict the agglomeration of dry ice particles in the tube.

\subsection{Distribution of particles in the jet flow}

Particle size measurements were carried out at different radial positions in the jet flow ejected from the tube. The nozzle diameter was $0.1 \mathrm{~mm}$ and the tube diameter was $4 \mathrm{~mm}$. Fig. 13 shows the mass median diameter and the attenuation of the dry ice jet as a function of radial position, $r$. The mass median diameter in the central axis of the jet flow is about $110 \mu \mathrm{m}$, and slightly decreases with the increase of the radial position, $r$, at $d_{\mathrm{t}}=20$ and $60 \mathrm{~mm}$ (see Fig. 13a). For $d_{\mathrm{t}}=60 \mathrm{~mm}$ and $r=18 \mathrm{~mm}$, the 
mass median diameter decreases to about $80 \mu \mathrm{m}$. As the jet flow expands downstream, larger agglomerates go straight along the jet axis because of their inertia, while smaller agglomerates spread in the radial direction. In addition, since the exterior of the jet is exposed to a greater rate of heat transfer with atmosphere, the agglomerates would sublimate at a faster rate with increased radial distance. As a result, the profile as a function of the radius of the jet flow will deviate from a flat distribution with increasing flow distance. The attenuation of the dry ice jet shows that the profile expands radially along the flow direction (see Fig. 13b.). The agglomerates are shown to be well dispersed in the jet flow. However, if the distance from the tube end is large, the effect of sublimation will be noticeable.

\section{Conclusions}

The size distribution and attenuation of the dry ice particles produced by rapid expansion of liquid carbon dioxide were analyzed online, downstream of the nozzle or the tube by a laser diffraction method. The conclusions were drawn as follows:

(1) With the experimental setup used in this work, the mass median diameter of the dry ice particles ejected from the expansion nozzle is about $1 \mu \mathrm{m}$. The particle size distribution is roughly log-normal. The particles grow in the jet flow and in the tubes. However, the sublimation of dry ice also occurs, and becomes noticeable at a distance from the nozzle outlet, where the particle size starts to reduce. As a result of the simultaneous phenomena of particle growth and sublimation, a bimodal size distribution exists at greater distances, arising from the size distributions of the primary particles and the agglomerated ones. For smaller expansion nozzles sublimation occurs at a shorter distance from the nozzle outlet, because the reduced flow rate results in a more rapid increase in temperature of the jet flow due to heat exchange with the atmosphere.

(2) Under the prevailing aerodynamic conditions, the primary particles agglomerate to about $100 \mu \mathrm{m}$ in mass median diameter by passing through the tube attached to the outlet of the nozzle. The mass median diameter of the agglomerates is not very sensitive to the nozzle diameter and varies from $100 \mu \mathrm{m}$ to $80 \mu \mathrm{m}$ when the latter is changed from 0.1 to $0.5 \mathrm{~mm}$. 
(3) A simple account of interparticle collisions in the tubes showed that most of the primary particles cannot agglomerate due to a collision mechanism in the short residence time within the tube.

(4) The agglomeration process is considered to be by particle deposition on the tube wall and reentrainment. Primary particles deposit on the tube wall and form a deposition layer; then, agglomerates are entrained from the deposition layer by aerodynamic drag and the collision of the flowing particles. The particle deposition and reentrainment reach an equilibrium rate if the process is operated sufficiently long to reach a steady state. The mass median diameter tends to decrease with the increase in the flow velocity. This is because the detachment force acting on the deposition layer increases with the flow velocity, smaller agglomerates can therefore be reentrained.

(5) As the jet flow extends radially downstream, larger agglomerates travel along the jet axis, while smaller agglomerates spread out in the radial direction, following more easily the gas streamlines. As a result, the profile as a function of the radius of the jet flow deviates from a flat distribution with increasing flow distance.

\section{Acknowledgements}

The authors acknowledge financial support by Core-to-Core Program for Advanced Particle Handling Science, JSPS. This research was also supported by the Global COE Program (B-09) from MEXT, Japan.

\section{References}

Adhiwidjaja, I., Matsusaka, S., Tanaka, H., \& Masuda, H. (2000). Simultaneous phenomenon of particle deposition and reentrainment: effects of surface roughness on deposition layer of striped pattern. Aerosol Science and Technology, 33, 323-333.

Friedlander, S. K. (2000). Collision and Coagulation. Smoke, Dust, and Haze (2nd ed.), Oxford: Oxford University, pp. 188-221. 
Harvill, T. L., Hoog, J. H., \& Holve, D. J. (1995). In-process particle size distribution measurements and control. Particle \& Particle Systems Characterization, 12, 309-313.

Harvill, T. L. \& Holve, D. J. (1998). Method for measuring particle size in the presence of multiple scattering. E.P. patent, 0781986-B1.

Hoenig, S. A. (1986). Cleaning surfaces with dry ice. Compressed Air Magazine, 91(8) $22-25$.

Liu, Y. H., Maruyama, H., \& Matsusaka, S. (2010). Agglomeration process of dry ice particles produced by expanding liquid carbon dioxide. Advanced Powder Technology, 21, 652-657.

Matson, D. W., \& Smith, R. D. (1989). Supercritical fluid technologies for ceramic-processing applications. Journal of the American Ceramic Society, 72, 871-881.

Matsusaka, S., \& Masuda, H. (1996). Particle reentrainment from a fine powder layer in a turbulent air flow. Aerosol Science and Technology, 24, 69-84.

Sherman, R., Hirt, D., \& Vane, R. (1994). Surface cleaning with the carbon dioxide snow jet. Journal of Vacuum Science \& Technology A, 12, 1876-1881.

Sonoda, R., Hara, Y., Iwasaki T., \& Watano, S. (2009). Improvement of dissolution property of poorly water-soluble drug by supercritical freeze granulation. Chemical \& Pharmaceutical Bulletin, 57, 1040-1044.

Swain, E. A., Carter, S. R., \& Hoenig, S. A. (1992). Carbon dioxide snow agglomeration and acceleration. U.S. Patent, 5125979. 
Theerachaisupakij, W., Matsusaka, S., Akashi, Y., \& Masuda, H. (2003). Reentrainment of deposited particles by drag and aerosol collision. Journal of Aerosol Science, 34, 261-274.

Yamaguchi, H., Zhang, X. R., \& Fujima, K. (2008). Basic study on new cryogenic refrigeration using $\mathrm{CO}_{2}$ solid-gas two phase flow. International Journal of Refrigeration, 31, 404-410. 


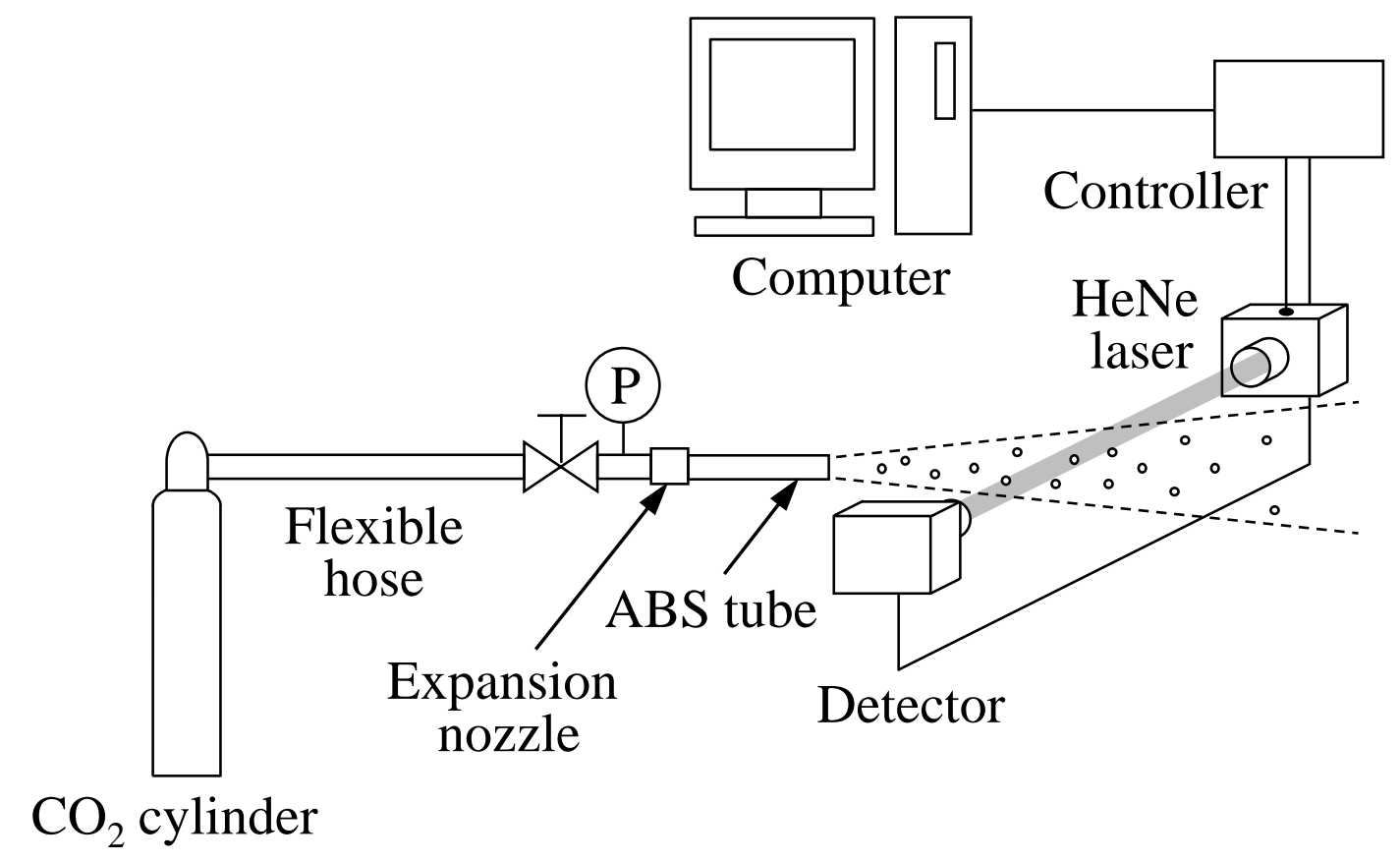

Fig. 1. Schematic diagram of experimental apparatus. 


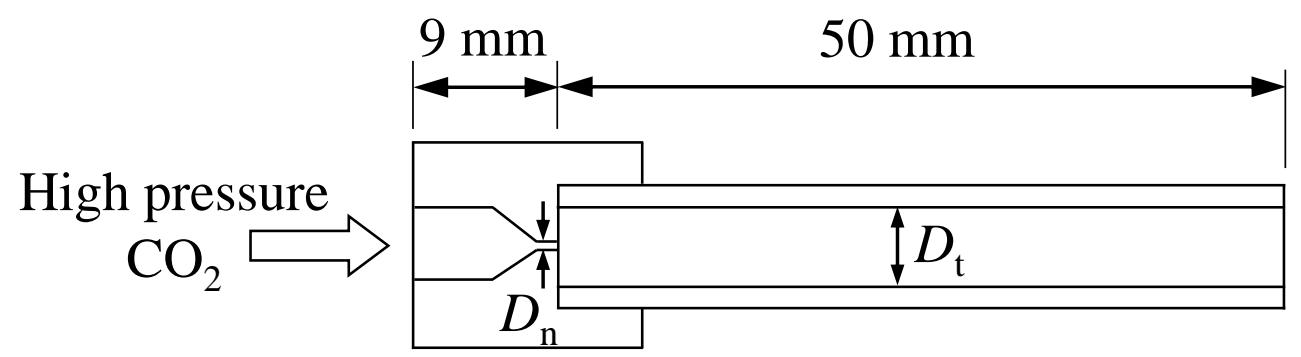

Expansion nozzle Removable ABS tube

$\left(D_{\mathrm{n}}=0.1,0.2,0.5 \mathrm{~mm}\right) \quad\left(D_{\mathrm{t}}=2,4,6 \mathrm{~mm}\right)$

Fig. 2. Cross-section of an expansion nozzle and a tube. 


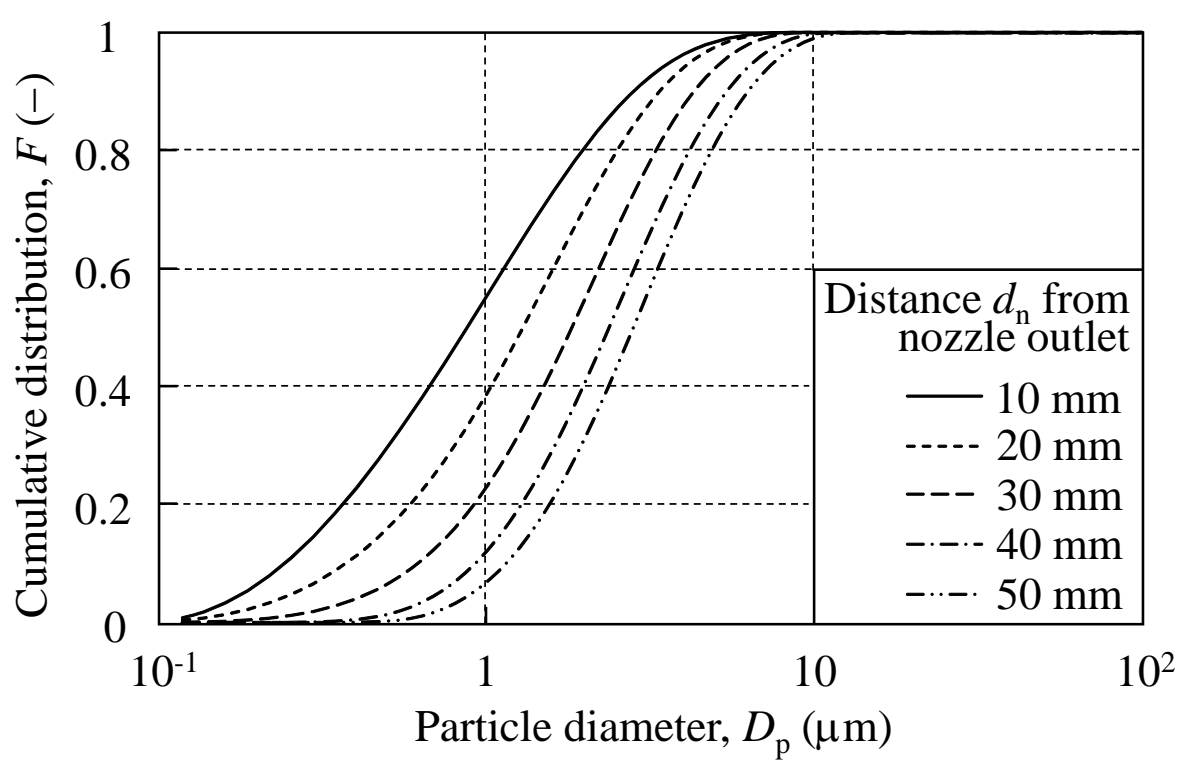

Fig. 3. Cumulative size distributions of dry ice particles ejected from expansion nozzle (nozzle diameter $D_{\mathrm{n}}=0.5 \mathrm{~mm}$ ). 


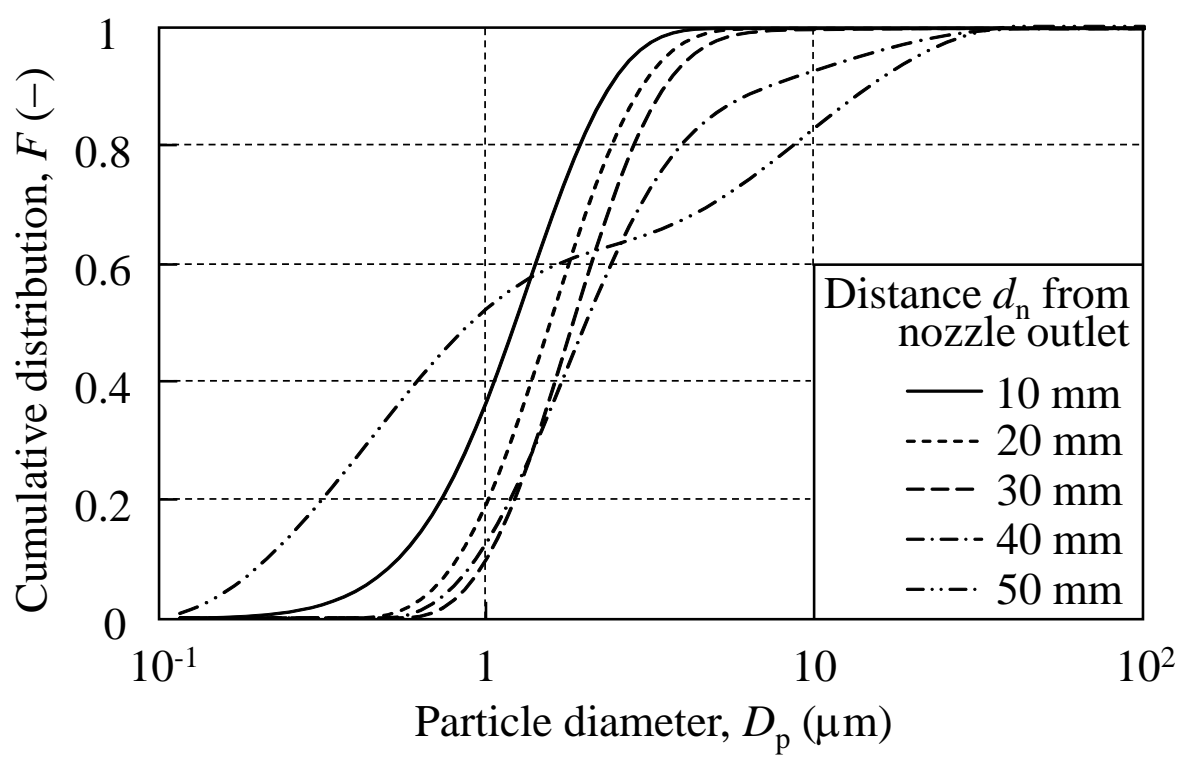

Fig. 4. Cumulative size distributions of dry ice particles ejected from expansion nozzle (nozzle diameter $D_{\mathrm{n}}=0.2 \mathrm{~mm}$ ). 


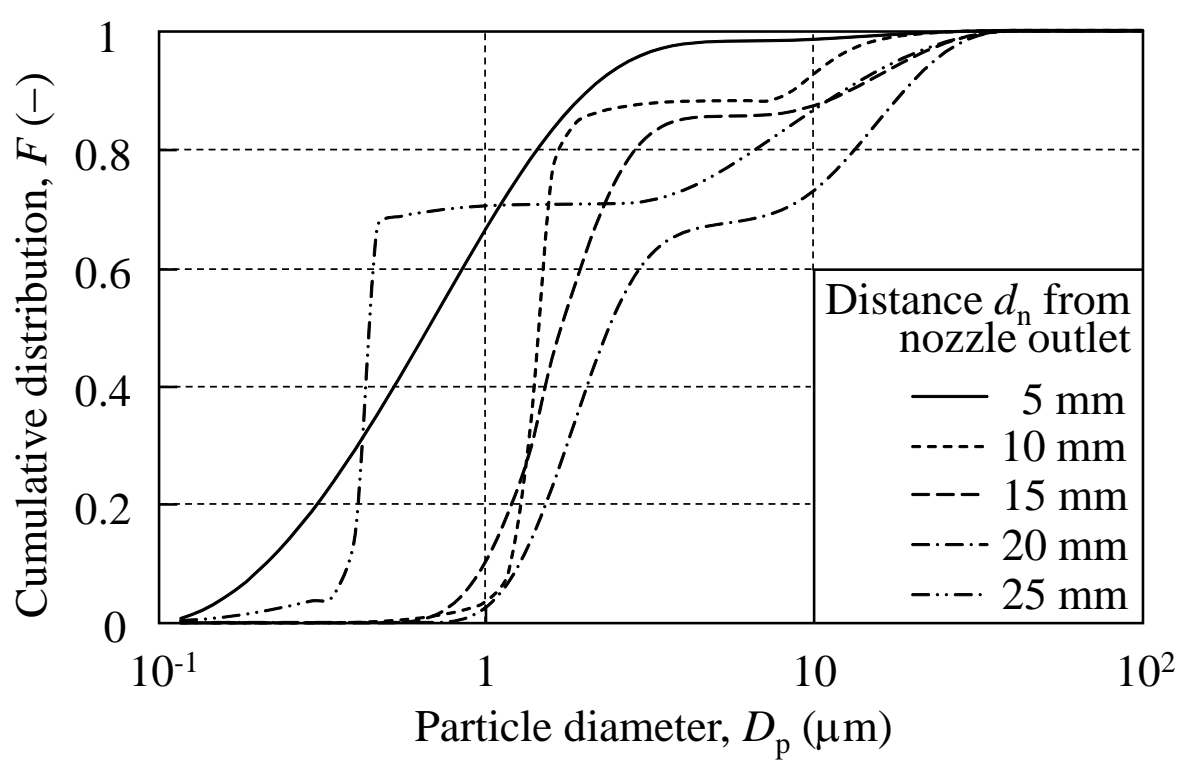

Fig. 5. Cumulative size distributions of dry ice particles ejected from expansion nozzle (nozzle diameter $D_{\mathrm{n}}=0.1 \mathrm{~mm}$ ). 

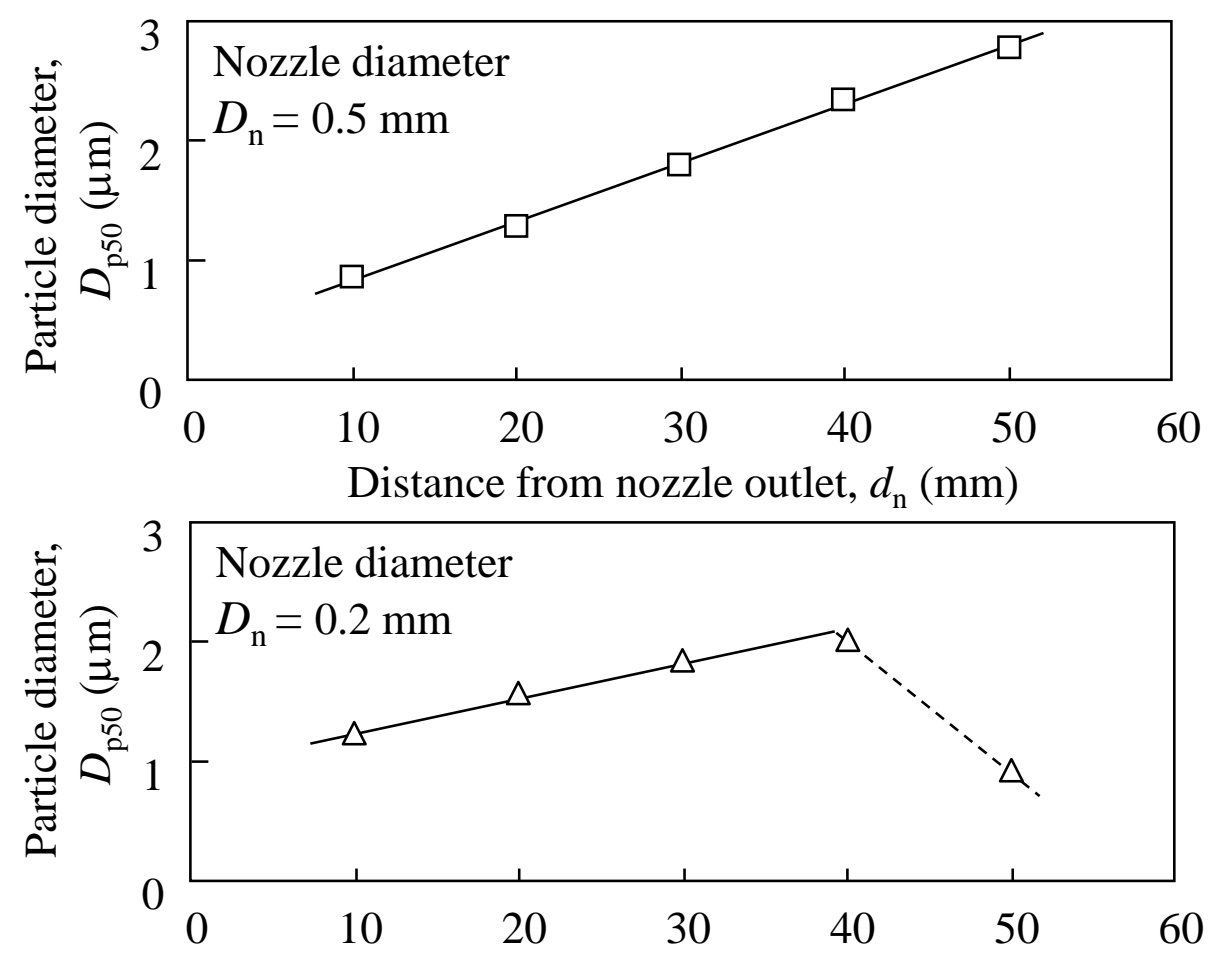

Distance from nozzle outlet, $d_{\mathrm{n}}(\mathrm{mm})$

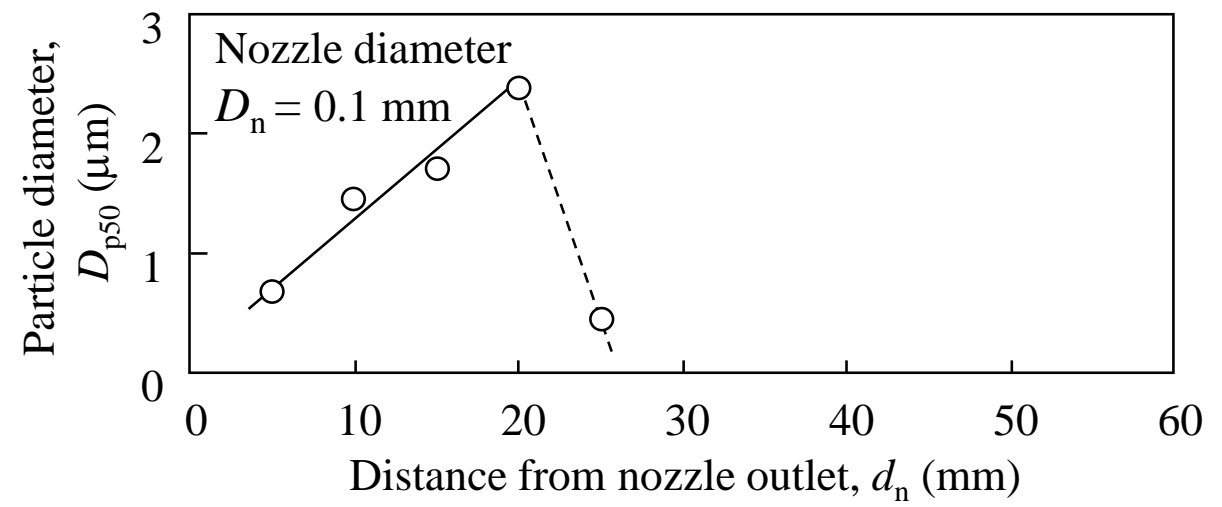

Fig. 6. Effect of nozzle diameter on mass median diameter of dry ice particles. 


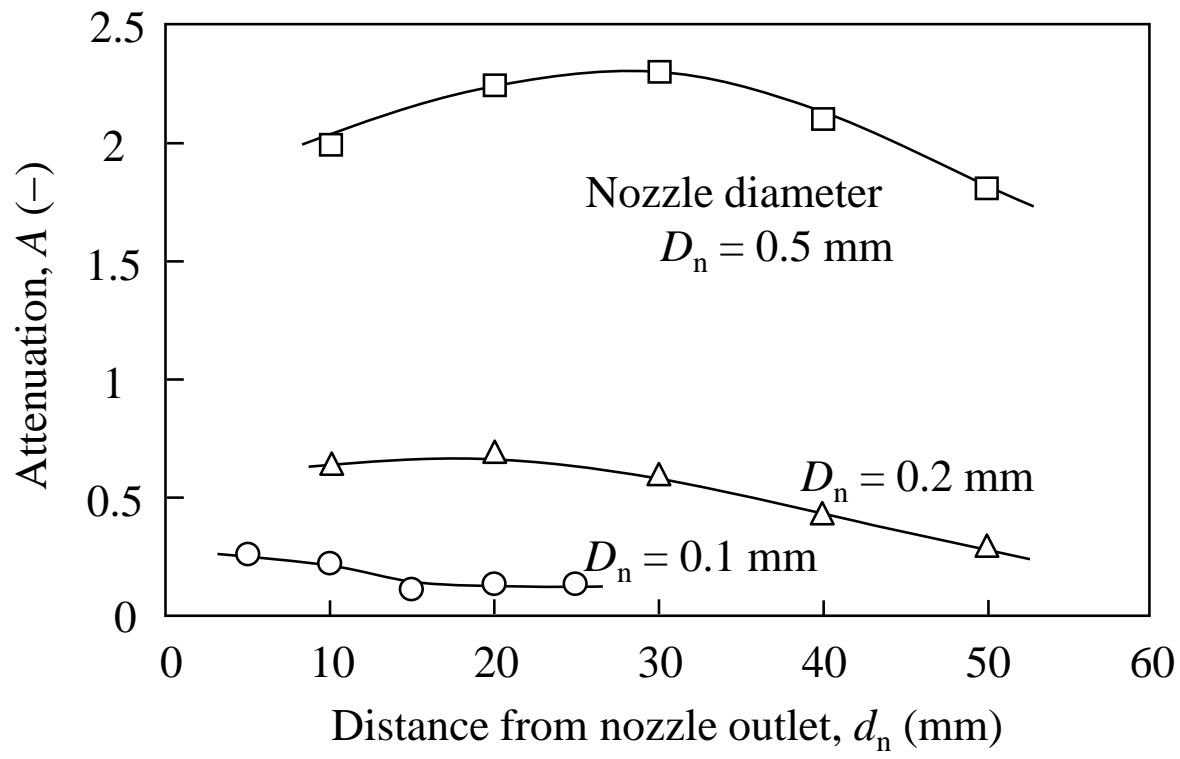

Fig. 7. Attenuation of dry ice jet ejected from expansion nozzle. 


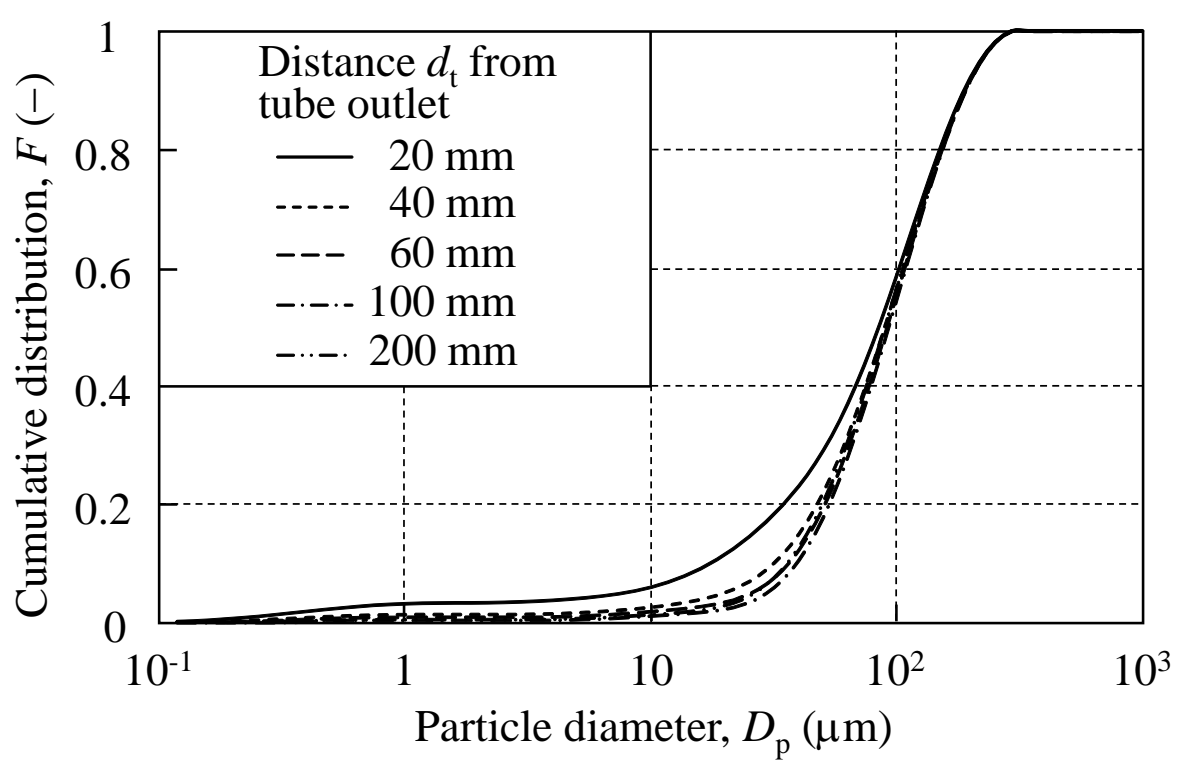

Fig. 8. Cumulative size distribution of dry ice particles ejected from tube (nozzle diameter $D_{\mathrm{n}}=0.5 \mathrm{~mm}$, tube diameter $D_{\mathrm{t}}=6 \mathrm{~mm}$ ). 


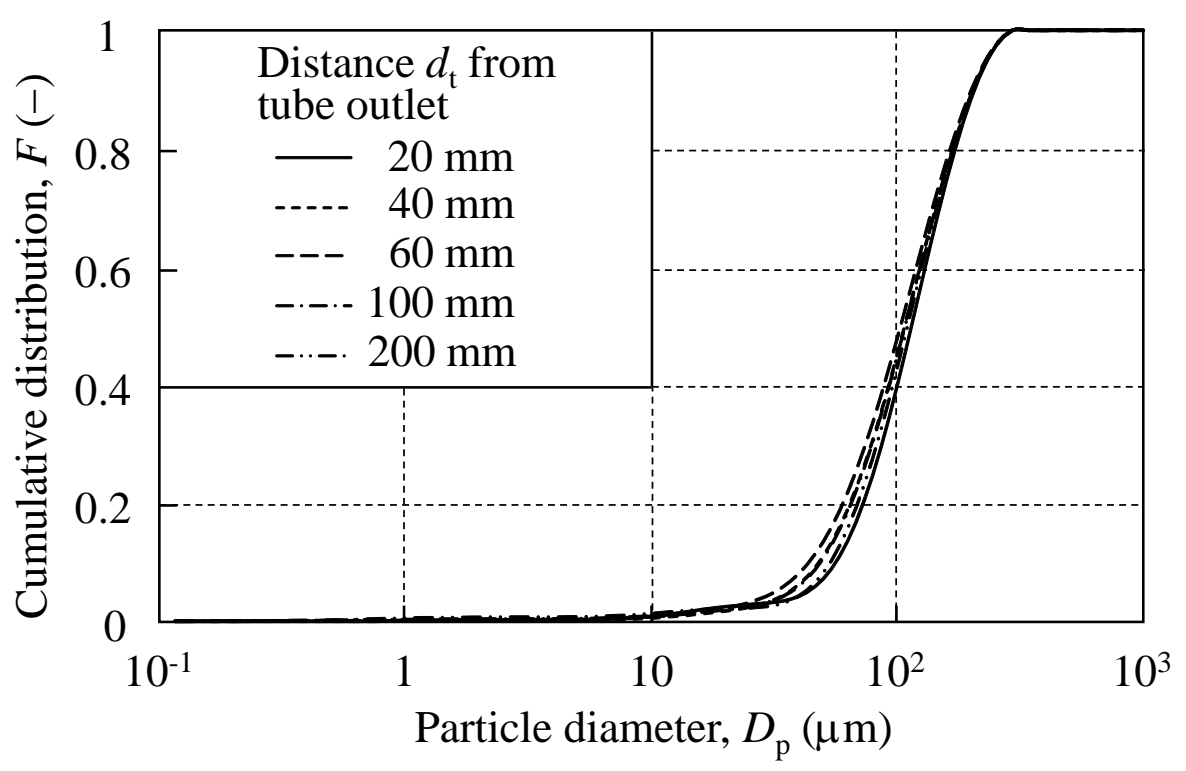

Fig. 9. Cumulative size distribution of dry ice particles ejected from tube (nozzle diameter $D_{\mathrm{n}}=0.1 \mathrm{~mm}$, tube diameter $D_{\mathrm{t}}=2 \mathrm{~mm}$ ). 


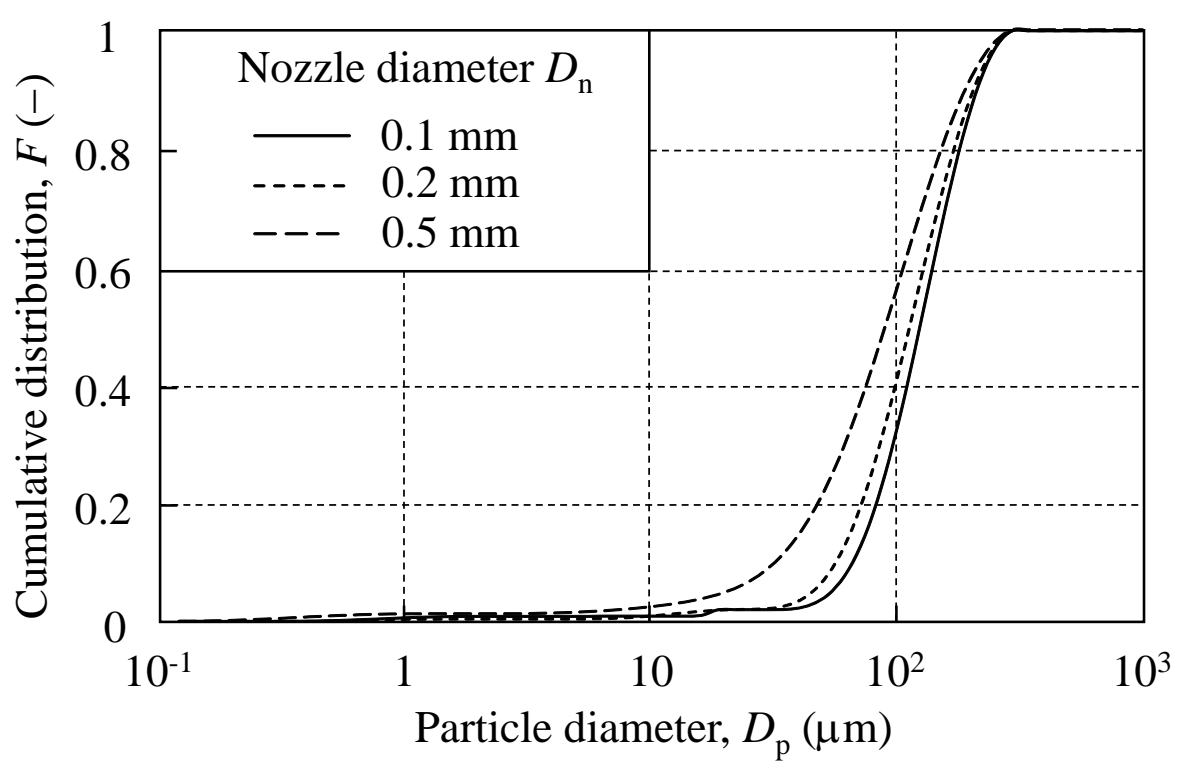

Fig. 10. The effect of nozzle diameter on cumulative size distribution of dry ice particles ejected from tube $\left(D_{\mathrm{t}}=6 \mathrm{~mm}, d_{\mathrm{t}}=40 \mathrm{~mm}\right)$. 


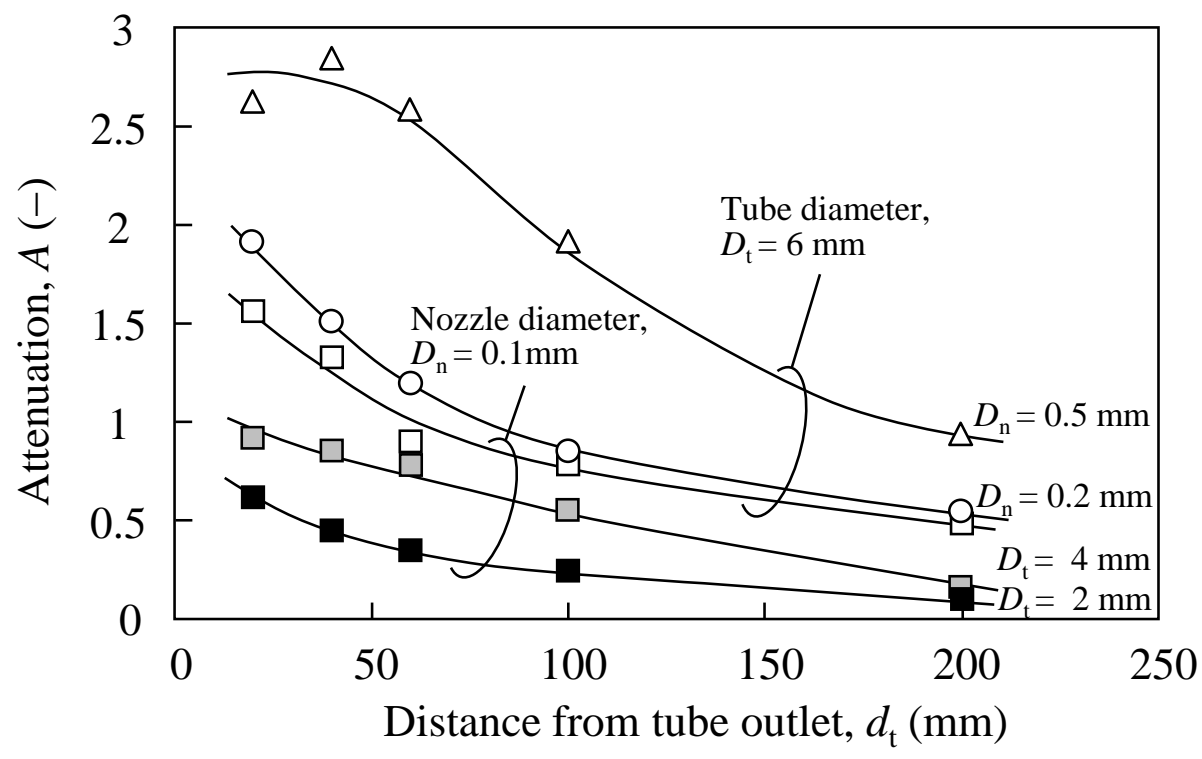

Fig. 11. Attenuation of dry ice jet ejected from tube. 


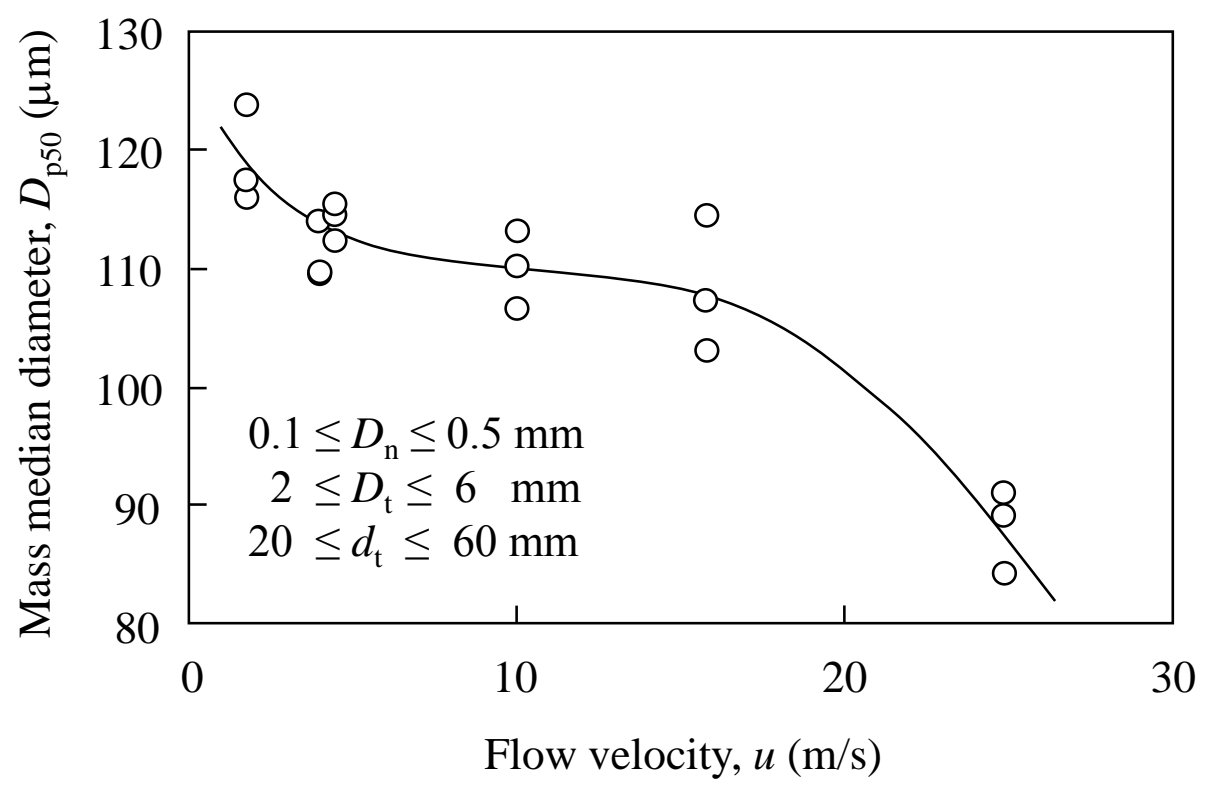

Fig. 12. Effect of flow velocity on mass median diameter of agglomerated dry ice particles. 

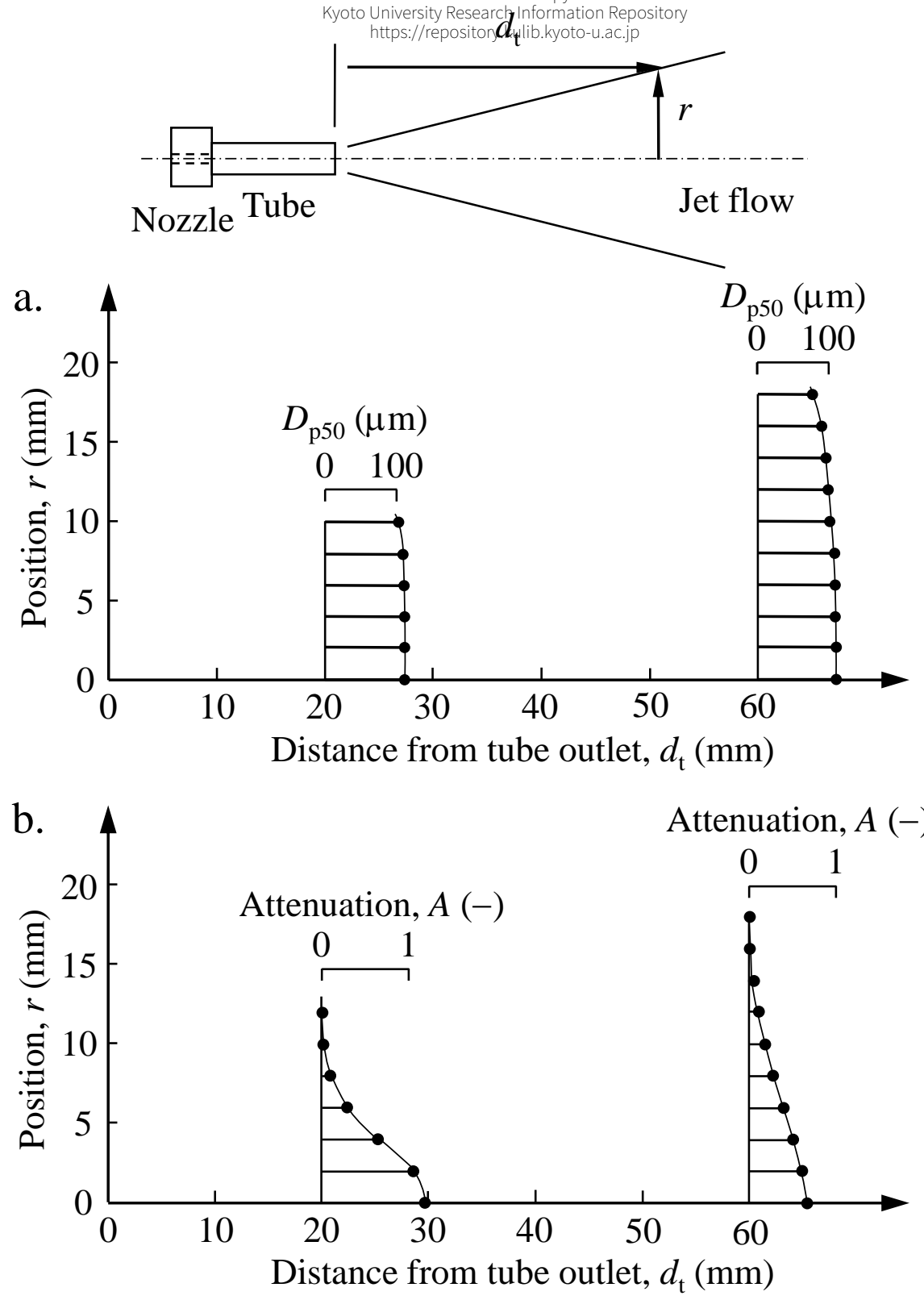

Fig. 13. Distribution of dry ice particles in jet flow: a. mass median diameter, $b$. attenuation $\left(D_{\mathrm{n}}=0.1 \mathrm{~mm}, D_{\mathrm{t}}=4 \mathrm{~mm}\right)$. 